\title{
Serum Lactate Dehydrogenase Contributes to Prognostic Assessment in Patients With Oligometastatic Cancer and Brain Involvement
}

\author{
CARSTEN NIEDER ${ }^{1,2}$, ASTRID DALHAUG $^{1}$ and ADAM PAWINSKI ${ }^{1}$ \\ ${ }^{1}$ Department of Oncology and Palliative Medicine, Nordland Hospital, Bod, , Norway; \\ ${ }^{2}$ Department of Clinical Medicine, Faculty of Health Sciences, \\ UiT - The Arctic University of Norway, Troms $\phi$, Norway
}

\begin{abstract}
Background/Aim: The aim of this study was to analyze the prognostic impact of serum lactate dehydrogenase $(L D H)$ in patients with oligometastatic brain metastases, arbitrarily defined as max. Four brain lesions and 5 metastatic lesions overall. Patients and Methods: This was a retrospective single institution analysis. Overall, 42 patients were identified from a prospectively maintained database. Results: Seventeen patients (40\%) had extracranial metastases. Twelve patients (29\%) had elevated $L D H(\geq 255 \mathrm{U} / \mathrm{l})$. Their median survival was significantly shorter than that of patients with normal LDH. Due to an interaction with performance status, this result was separately confirmed in patients with performance status $\geq 70$. Conclusion: Oligometastatic disease is not always correctly diagnosed, because all radiological modalities are limited by certain thresholds for detection of small metastases. We hypothesize that $\mathrm{LDH}$ is associated with survival, because this biomarker may reflect the total burden of malignant disease. Future studies should examine whether or not ablative local treatment of oligometastases is warranted in patients with elevated $\mathrm{LDH}$.
\end{abstract}

Increasing attention has recently been paid to cancer patients with oligometastatic disease, i.e. a state between curable locoregional disease extension and incurable dissemination throughout large parts of the body (1-4). If only few distant metastases are present, ablative local treatment and/or

This article is freely accessible online.

Correspondence to: Dr. Carsten Nieder, Department of Oncology and Palliative Medicine, Nordland Hospital, 8092 Bod $\varnothing$, Norway. Tel: +47 75578449, Fax: +47 75534975, e-mail: carsten.nieder@nlsh.no

Key Words: Palliative radiotherapy, whole brain radiotherapy, brain metastases, oligometastases, prognostic factors, lactate dehydrogenase. resection of all these lesions might result in longer survival than historically observed (5-8). However, not all patients become long-term survivors, a finding that emphasizes the challenges associated with detecting true oligometastatic disease. If additional micrometastases go undetected, rapid disease progression might be encountered. It would, therefore, be prudent to include staging examinations that are not limited by the detection thresholds of radiological methods $(9,10)$. For example, the number of circulating tumor cells or other emerging biomarkers may aid in the detection of true and pseudo-oligometastatic disease $(11,12)$. Given that many patients, especially in low- and middle-income countries, lack access to advanced imaging and biomarker evaluations, widely available and inexpensive biomarkers may also contribute to improved prognostic assessment and decisionmaking. Based on our previous work on the prognostic impact of serum lactate dehydrogenase (LDH) in patients with brain metastases (13), we hypothesized that LDH might reflect overall disease burden also in patients presumed to harbor oligometastases. If true, patients with elevated LDH due to otherwise undetectable additional metastases are expected to have poorer survival and may spared the burden of aggressive local treatment. This hypothesis was tested in a previously utilized, continuously updated database $(13,14)$.

\section{Materials and Methods}

Patients and treatment. A retrospective study of all patients with maximum 4 parenchymal brain metastases from histologically verified extracranial primary tumors treated with whole-brain radiotherapy (WBRT) at our hospital was performed. Further treatment was individualized and consisted of salvage radiosurgery (SRS), systemic therapy or best supportive care (BSC). Systemic treatment was usually prescribed as judged appropriate by the patients' medical oncologists. The patients were treated between January 01, 2007 and December 31, 2017. Brain magnetic resonance imaging (MRI) was performed to assess the number of intracranial metastases and to exclude leptomeningeal disease. 
Table I. Patient characteristics.

\begin{tabular}{lrc}
\hline Baseline parameter & Number & Percent \\
\hline Non-small cell lung cancer & 24 & 57 \\
Small cell lung cancer & 9 & 21 \\
Breast cancer & 6 & 14 \\
Melanoma & 3 & 7 \\
Extracranial metastases & 17 & 40 \\
Female gender & 18 & 43 \\
Male gender & 24 & 57 \\
One brain metastasis & 16 & 38 \\
Two or three brain metastases & 21 & 50 \\
Four brain metastases & 5 & 12 \\
Elevated lactate dehydrogenase & 12 & 29 \\
KPS 90-100 & 2 & 5 \\
KPS 80 & 10 & 24 \\
KPS 70 & 15 & 36 \\
KPS <70 & 15 & 36 \\
RPA class 1 & 4 & 10 \\
RPA class 2 & 23 & 55 \\
RPA class 3 & 15 & 36 \\
\hline
\end{tabular}

KPS: Karnofsky performance status; RPA: recursive partitioning analysis.

Extracranial staging consisted of computed tomography (CT). Patients with extracranial metastases to one organ system, e.g., adrenal glands or distant lymph nodes (beyond regional drainage), were included as long as the total number of metastases extra- and intracranially was limited to five or fewer. LDH was assessed at the start of WBRT or within the preceding week (the normal value was defined as $<255 \mathrm{U} / \mathrm{l})$.

Statistical methods. Overall survival (time to death) from the first day of radiotherapy was calculated employing the Kaplan-Meier method, and different groups were compared using the log-rank test (SPSS 25, IBM Corp., Armonk, NY, USA). Only one patient was censored after 49 months of follow-up. Date of death was known in all other patients. A Cox forward conditional regression model was employed for multivariate analysis. Statistical significance was defined as $p<0.05$ throughout this study.

\section{Results}

Patient characteristics. We identified 42 patients from the database. Twenty-four $(57 \%)$ had non-small cell lung cancer (NSCLC). Seventeen patients $(40 \%)$ had extracranial metastases and the others brain-only dissemination. The median age was 68 years (range $=28-90$ years). Further patient characteristics are shown in Table I.

Treatment. Eighteen patients $(43 \%)$ received systemic treatment. The typical WBRT regimen was 10 fractions of 3 Gy. Extracranial metastases were not treated with local measures such as surgery or ablative radiotherapy.

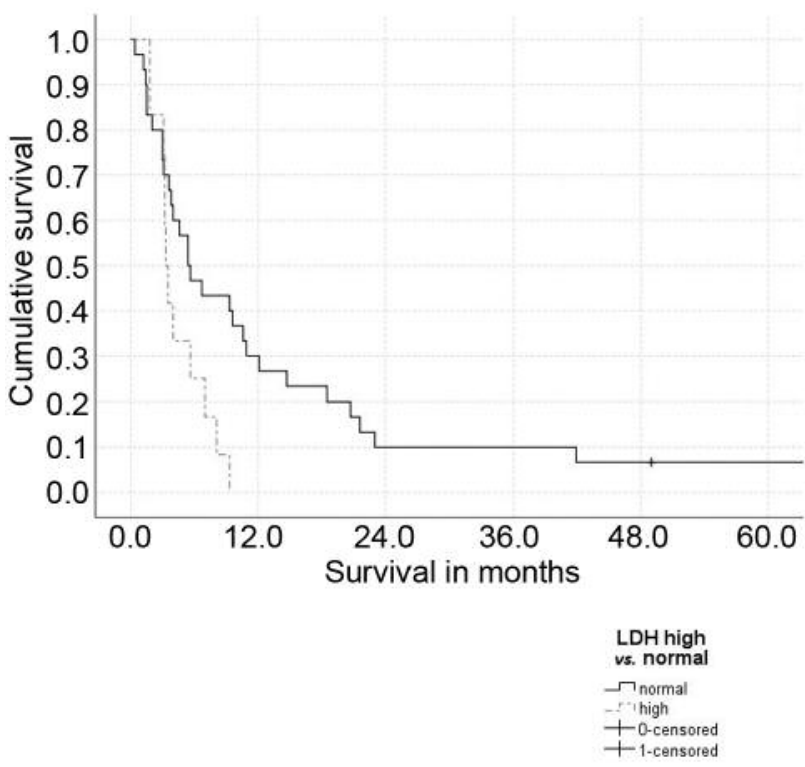

Figure 1. Actuarial overall survival stratified by serum lactate dehydrogenase (normal LDH: median 5.4 months, elevated LDH: median 3.3 months, $p=0.03$ ).

Survival. The median overall survival of all patients was 5.4 months (21\% 1-year survival). As shown in Figure 1, LDH was significantly associated with survival. Survival beyond one year was observed in patients with normal LDH only. Age, number of brain metastases, and presence of extracranial metastases were not significantly associated with elevated LDH. However, patients with elevated LDH were more likely to have reduced performance status and higher recursive partitioning analysis class $(p=0.09$, Chi-square test). Performance status was more important in the multivariate analysis than $\mathrm{LDH}$, number of brain metastases and presence of extracranial metastases $(p=0.03$ compared to $>0.4$ for the three other variables). When excluding patients with performance status $<70$ both log-rank test and Cox regression analysis showed that $\mathrm{LDH}$ was the only significant predictor of survival ( $p=0.03$ in the multivariate model with all four variables).

\section{Discussion}

We performed a retrospective study of patients with a limited number of brain metastases (between 1 and 4) and a maximum number of five metastases in total, which involved only one extracranial organ system, e.g., bilateral adrenal gland metastases. Sixty percent had brain-only metastases. We found that elevated LDH was associated with significantly shorter survival, in line with our previous results in a larger study of patients who mainly had non- 
oligometastatic brain metastases (13) and with other studies in different settings (15-17).

Limitations of the present study include the limited number of patients, statistical power of subgroup analyses, and retrospective design. The presence of oligometastatic disease was not confirmed by positron emission tomography (PET) staging. However, PET staging is also associated with falsenegative and otherwise inaccurate findings (10). Moreover, PET capacity is limited or in some low- and middle-income countries non-existent. Under these circumstances, readilyavailable biomarkers might complement CT-based staging with the ultimate goal of avoiding overtreatment in patients with pseudo-oligometastatic disease. Emerging biomarkers such as circulating tumor cells are not yet widely available, in contrast to LDH measurement.

We limited this analysis to patients treated with primary WBRT in order to eliminate bias from differences in local treatment. If some patients receive SRS, which results in superior local control $(18,19)$, survival is no longer a function of baseline prognostic parameters alone. As recently shown, aggressive local treatment of oligometastatic cancer appears to improve survival $(5,20)$. Compared to published survival outcomes from studies employing more effective local treatment our results are relatively disappointing $(21 \%$ 1-year survival). In part, this can also be explained by the selection of patients who were treated with primary WBRT and the fact that patients with reduced performance status were included. Notably, we found long-term survivors in the group with normal LDH despite the absence of ablative local treatment. Our results suggest that assessment of LDH may add value when designing further clinical trials and also in resource-limited settings.

\section{Conclusion}

Oligometastatic disease is not always correctly diagnosed, because all radiological modalities are limited by certain thresholds for detection of small metastases. We hypothesize that LDH is associated with survival, because this biomarker may reflect the total burden of malignant disease. Future studies should examine whether or not ablative local treatment of oligometastases is warranted in patients with elevated LDH.

\section{Conflicts of Interest}

The Authors declare that they have no competing interests.

\section{References}

1 Tosoian JJ, Gorin MA, Ross AE, Pienta KJ, Tran PT and Schaeffer EM: Oligometastatic prostate cancer: definitions, clinical outcomes, and treatment considerations. Nat Rev Urol 14: 15-25, 2017.

2 Nieder C, Tollåli T, Reigstad A, Pawinski A, Haukland E and Dalhaug A: Oligometastatic non-small cell lung cancer: a significant entity outside of specialized cancer centers? Med Princ Pract 23: 526-531, 2014.

3 Ashworth AB, Senan S, Palma DA, Riquet M, Ahn YC, Ricardi U, Congedo MT, Gomez DR, Wright GM, Melloni G, Milano MT, Sole CV, De Pas TM, Carter DL, Warner AJ and Rodrigues GB: An individual patient data metaanalysis of outcomes and prognostic factors after treatment of oligometastatic non-smallcell lung cancer. Clin Lung Cancer 15: 346-355, 2014.

4 Palma DA, Louie AV and Rodrigues GB: New strategies in stereotactic radiotherapy for oligometastases. Clin Cancer Res 21: 5198-5204, 2015.

5 Gomez DR, Blumenschein GR Jr, Lee JJ, Hernandez M, Ye R, Camidge DR, Doebele RC, Skoulidis F, Gaspar LE, Gibbons DL, Karam JA, Kavanagh BD, Tang C, Komaki R, Louie AV, Palma DA, Tsao AS, Sepesi B, William WN, Zhang J, Shi Q, Wang XS, Swisher SG and Heymach JV: Local consolidative therapy versus maintenance therapy or observation for patients with oligometastatic non-small-cell lung cancer without progression after first-line systemic therapy: a multicentre, randomised, controlled, phase 2 study. Lancet Oncol 17: 1672$1682,2016$.

6 Pasqualetti F, Panichi M, Sainato A, Baldaccini D, Cocuzza P, Gonnelli A, Montrone S, Molinari A, Barbiero S, Bruschi A, Notini E, Ursino S, Mazzotti V, Morganti R, Coraggio G, Cantarella M, Erba PA and Paiar F: Image-guided stereotactic body radiotherapy in metastatic prostate cancer. Anticancer Res 38: 3119-3122, 2018.

7 Ampil F, Ellika S, Nanda A and Vora M: Long-term survival after stereotactic radiosurgery of brain metastases: A case series with 10-year follow-up. Anticancer Res 37: 5113-5115, 2017.

8 Pasqualetti F, Montrone S, Vivaldi C, Zani M, Fedele D, Fornaro L, Pasqualetti G, Salvatore L, Manfredi B, Laliscia C, Coraggio G, Gonnelli A, Loupakis F, Masi G, Sainato A, Monzani F, Falcone A and Paiar F: Stereotactic body radiotherapy in patients with lung oligometastases from colorectal cancer. Anticancer Res 37: 315-319, 2017.

9 Lecouvet FE, Oprea-Lager DE, Liu Y, Ost P, Bidaut L, Collette L, Deroose CM, Goffin K, Herrmann K, Hoekstra OS, Kramer G, Lievens Y, Lopci E, Pasquier D, Petersen LJ, Talbot JN, Zacho H, Tombal B and deSouza NM: Use of modern imaging methods to facilitate trials of metastasis-directed therapy for oligometastatic disease in prostate cancer: a consensus recommendation from the EORTC Imaging Group. Lancet Oncol 19: e534-e545, 2018.

10 deSouza NM, Liu Y, Chiti A, Oprea-Lager D, Gebhart G, Van Beers BE, Herrmann $\mathrm{K}$ and Lecouvet FE: Strategies and technical challenges for imaging oligometastatic disease: Recommendations from the European Organisation for Research and Treatment of Cancer imaging group. Eur J Cancer 91: 153$163,2018$.

11 Yang C, Shi D, Wang S, Wei C, Zhang C and Xiong B: Prognostic value of pre- and post-operative circulating tumor cells detection in colorectal cancer patients treated with curative resection: a prospective cohort study based on ISET device. Cancer Manag Res 10: 4135-4144, 2018.

12 Tinhofer I and Staudte S: Circulating tumor cells as biomarkers in head and neck cancer: recent advances and future outlook. Expert Rev Mol Diagn 18: 897-906, 2018.

13 Nieder C, Marienhagen K, Dalhaug A, Aandahl G, Haukland E and Pawinski A: Prognostic models predicting survival of 
patients with brain metastases: integration of lactate dehydrogenase, albumin and extracranial organ involvement. Clin Oncol (R Coll Radiol) 26: 447-452, 2014.

14 Nieder C, Hintz M, Oehlke O, Bilger A and Grosu AL: The TNM 8 M1b and M1c classification for non-small cell lung cancer in a cohort of patients with brain metastases. Clin Transl Oncol 19: 1141-1146, 2017.

15 Zhou L, Xie Z, Shao Z, Chen W, Xie H, Cui X, Qin G and Zhao $\mathrm{N}$ : Modeling the relationship between baseline lactate dehydrogenase and prognosis in patients with extensive-disease small cell lung cancer: a retrospective cohort study. J Thorac Dis 10: 1043-1049, 2018.

16 Miyazawa K, Shikama N, Okazaki S, Koyama T, Takahashi T and Kato S: Predicting prognosis of short survival time after palliative whole-brain radiotherapy. J Radiat Res 59: 43-49, 2018.

17 Nieder C, Dalhaug A, Pawinski A, Mannsåker B and Haukland E: Survival after palliative radiotherapy in patients with breast cancer and bone-only metastases. In Vivo 30: 879-883, 2016.
18 Rades D, Dziggel L and Schild SE: A specific survival score for patients receiving local therapy for single brain metastasis from a gynecological malignancy. In Vivo 32: 825-828, 2018.

19 Rades D, Blanck O, Khoa MT, VAN Thai P, Hung NQ, Dziggel $\mathrm{L}$ and Schild SE: Validation of a survival score for patients receiving radiosurgery or fractionated stereotactic radiotherapy for 1 to 3 brain metastases. In Vivo 32: 381-384, 2018.

20 Stenman M, Sinclair G, Paavola P, Wersäll P, Harmenberg U and Lindskog M: Overall survival after stereotactic radiotherapy or surgical metastasectomy in oligometastatic renal cell carcinoma patients treated at two Swedish centres 2005-2014. Radiother Oncol 127: 501-506, 2018.
Received October 30, 2018

Revised November 12, 2018

Accepted November 13, 2018 